\title{
СОВЕРШЕНСТВОВАНИЕ ТЕХНИКИ ХИРУРГИЧЕСКИХ ВМЕШАТЕЛЬСТВ ПРИ ЛЕЧЕНИИ ПАЦИЕНТОВ С ДОБРОКАЧЕСТВЕННОЙ ГИПЕРПЛАЗИЕЙ ПРЕДСТАТЕЛЬНОЙ ЖЕЛЕЗЫ (ЛИТЕРАТУРНЫЙ ОБЗОР)
}

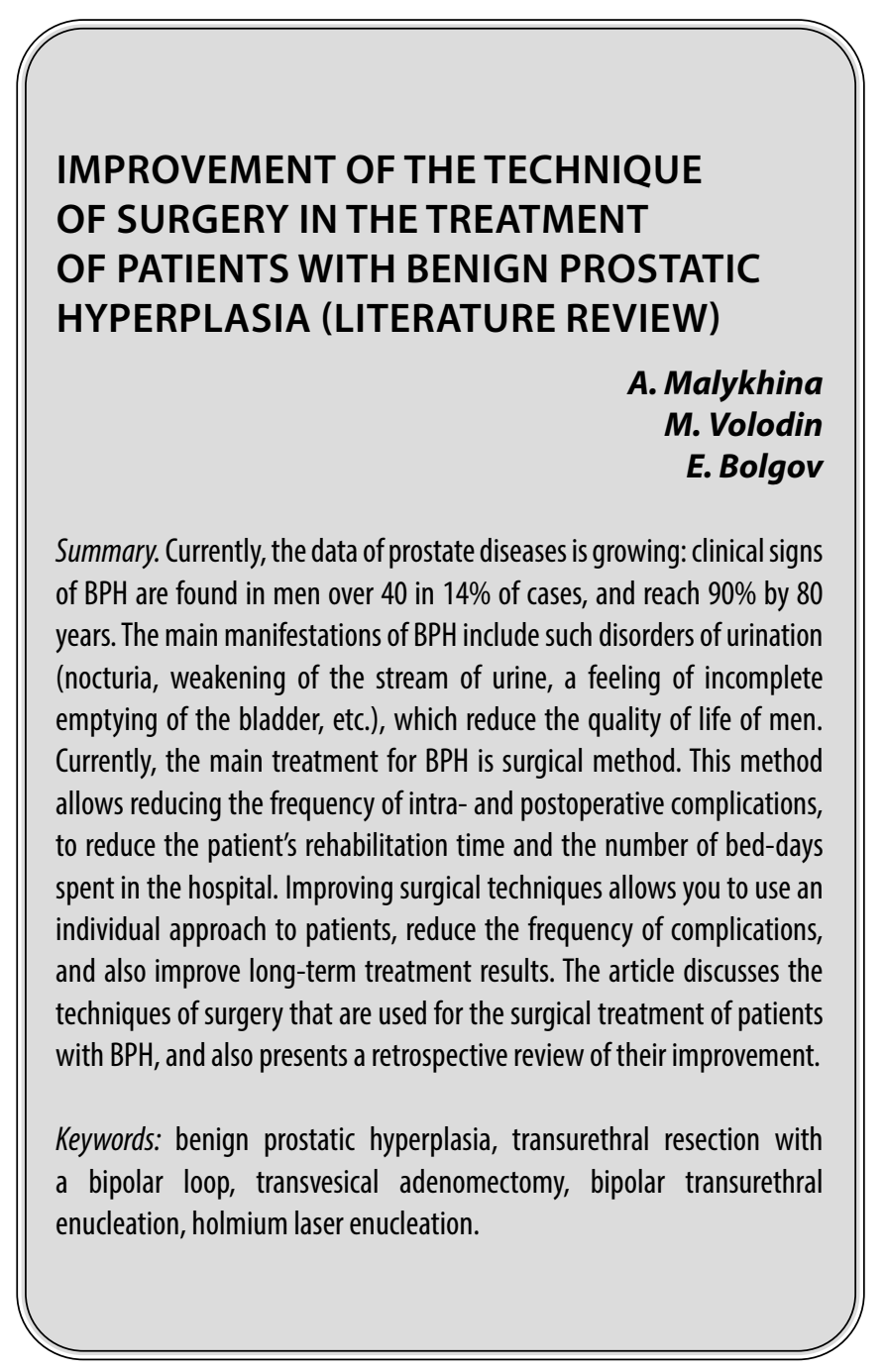

\section{Введение}

Д оброкачественная гиперплазия предстательной железы (ДГПЖ) является самым распространенным заболеванием мочеполовой системы у мужчин пожилого возраста. С каждым годом распространенность заболевания увеличивается, что связано как с возрастанием доли пожилых мужчин за счет увеличения продолжительности жизни, так и с улучшением диагностики, которое позволяет обнаруживать ДГПЖ уже на ранних стадиях $[3,10]$. Статистические данные утверждают, что клинические признаки ДГПЖ проявляются у $14 \%$ мужчин в возрасте от 40 до 49 лет, у 24\% мужчин

\author{
Малыхина Анастасия Сергеевна \\ ФГБОУ ВО ПИМУ Минздрава России, г. Нижний \\ Новгород \\ Володин Марк Альбертович \\ Аспирант, ФГБОУ ВО ПИМУ Минздрава России, \\ 2. Нижний Новгород \\ Болгов Евгений Николаевич \\ АНМО Ставропольский краевой клинический \\ консультативно-диагностический чентр, \\ 2. Ставрополь \\ peregal@yandex.ru
}

Аннотация. В настоящее время число заболеваний предстательной железы растет: клинические признаки ДГПЖ обнаруживаются у мужчин старше 40 лет в 14\% случаев, и достигают 90\% к 80 годам. К основным проявлениям аденомы предстательной железы относятся такие нарушения мочеиспускания (ноктурия, ослабление струи мочи, чувство неполного опорожнения мочевого пузыря и др.), которые снижают качество жизни мужчин. В настоящее время хирургический метод лечения ДГПЖ занимает отдельную нишу. Данный способ позволяет снизить частоту интра- и постоперационных осложнений, уменьшить время реабилитации пациента и количество койко-дней, проведенных в стационаре. Совершенствование хирургических методик позволяет использовать индивидуальный подход к пациентам, уменьшить частоту осложнений, а также улучшить отдаленные результаты лечения. В статье рассмотрены техники оперативных вмешательств, которые используются для хирургического лечения пациентов с ДГПЖ, а также представлен ретроспективный обзор их совершенствования.

Ключевые слова: доброкачественная гиперплазия предстательной железы, трансуретральная резекция биполярной петлей, чреспузырная аденомэктомия, биполярная трансуретральная энуклеация, гольмиевая лазерная энуклеация.

от 50 до 59 лет, у $43 \%$ от 60 до 69 лет, у $40 \%$ от 70 до 79 лет и у $90 \%$ мужчин старше 80 лет [8].

Патогенез и прогрессирование ДГПЖ до сих пор не изучены полностью, но по данным ряда авторов, заболевание является многофакторным с повышением активности симпатической нервной системы, гормональными изменениями, наличием метаболического синдрома и ремоделированием тканей, связанным со старением [20]. Симптомы нижних мочевых путей (СНМП), сопутствующие ДГПЖ, приводят к ухудшению качества жизни пациентов из-за недержания мочи, ноктурии, а также повышенного риска острой задержки мочи. Кроме того, СНМП являются фактором ри- 
ска для развития эректильной дисфункции [25]. Чрезмерное употребление красного мяса, насыщенных жиров, хлеба, птицы также способствует развитию СНМП [19].

Лечение ДГПж включает в себя как медикаментозную терапию, так и проведение хирургического вмешательства. Терапия а-адреноблокаторами является широко используемым и эффективным методом лечения для улучшения СНМП за счет расслабления гладкой мускулатуры предстательной железы и шейки мочевого пузыря [3]. На фоне лечения отмечается ослабление ирритативных симптомов, уменьшение гиперактивности детрузора. К побочным эффектам приема а-адреноблокаторов относят усталость, головокружение, ортостатическую гипотензию, проблемы с эякуляцией. Ингибиторы 5а-редуктазы (5АРИ) препятствуют преобразованию тестостерона в дигидротестостерон, который главным образом ответственен за гиперплазию и гипертрофию простаты. Несмотря на непрерывно пополняемый арсенал фармацевтических средств, хирургический метод лечения СНМП не теряет актуальности и занимает отдельную нишу [14, 23, 24].

Максимальное число оперативных вмешательств по поводу ДГПЖ приходится на долю мужчин в возрасте 50-70 лет, что связано с увеличением сопутствующих заболеваний в более позднем возрасте и успешным лечением заболевания консервативным путем в более молодом. Хирургическое вмешательство рекомендуется пациентам с неэффективностью медикаментозного лечения или при отказе от него, а также при нарастании СНМП или развитии осложнений ДГПж [13].

В настоящее время предпочтение отдается эндоскопическим хирургическим методам за счет меньшей травматичности, уменьшения послеоперационных осложнений, снижения количества дней, проведенных пациентом в стационаре, более короткого времени реабилитации [8]. Одним из наиболее распространенных показаний для проведения трансуретральной резекции простаты (ТУРП) является неудавшаяся медикаментозная терапия. [9, 28]. Таким образом, целью проведенного исследования стал ретроспективный обзор становления хирургических методов лечения Дгпж.

\section{Материалы и методы}

При проведении литературного поиска был использован научный архив и проанализированы следующие базы данных: PubMed, Springer, eLibrary, Cyberleninca.

\section{Обсужление}

Хирургическое лечение ДГПЖ зародилось в XIX веке. Первая операция была проведена во Франции в 1827 году хирургом Амюсса и представляла собой промежностную аденомэктомию. Начиная с 1848 года, Фергюссон, а чуть позже и некоторые другие хирурги, начал практиковать проведение эктомии предстательной железы во время промежностной литотомии. При этом мочеиспускание пациентов после операции улучшалось. Однако она проводились частично слепым методом. Концепция промежностной аденомэктомии под контролем зрения была описана Кушлером в 1866 г. и претворена в жизнь Бильротом в 1867 г. В начале XX века Янг модифицировал операцию, проводимую через зону каудальной простаты, тесно связанной с волокнами наружного сфинктера. Он разработал и внедрил в использование ретрактор, носящий его имя и сейчас [1].

Чреспузырный доступ вплоть до конца XIX века не был применен ни разу: хирурги избегали околопузырную область из-за высокой вероятности инфицирования. Лишь в 1899 году русский хирург С.П. Федоров, областью научных интересов которого являлись вопросы хирургического лечения ДГПЖ, впервые в мире провел типичную надлобковую аденомэктомию. Один из основоположников урологии в СССР, профессор Б.Н. Хольцов, продолжил развивать данное направление, разработав в том числе 2-х и 3-х моментный методы аденомэктомии [15]. В настоящее время открытые оперативные вмешательства по поводу ДГПЖ могут проводиться при больших объемах аденомы простаты $\left(>100 \mathrm{~cm}^{3}\right)$, наличии камней в мочевом пузыре. Количество проведенных чреспузырных аденомэктомий становится меньше в связи с высоким риском появления обструктивных (инфравезикальная обструкция, стриктура простатического отдела уретры) и воспалительных (эпидидимит, орхоэпидидимит, острый пиелонефрит) осложнений в раннем и позднем послеоперационном периоде [5]. Предрасполагающими факторами в данном случае служат: длительность хирургической операции, травматичность, длительное дренирование мочевого пузыря, а также способ гемостаза ложа простаты. Для профилактики развития осложнений рекомендуется раннее восстановление физиологического оттока мочи, которое можно достичь путем проведения одномоментной аденомэктомии и наложением глухого шва на мочевой пузырь, использования съемных лигатур на шейку мочевого пузыря для обеспечения гемостаза ложа аденомы простаты, проведения профилактической антибиотикотерапии [6].

Оперативная эндоскопия получила развитие благодаря Боттини, который в 1877 году разработал гальванокаутер - металлический наконечник, который накаливается путем прохождения через него электрического тока и, таким образом, коагулирует окружающие мягкие ткани. Однако главной сложностью использования данного инструмента являлась невозможность визуального наблюдения за ходом операции. Позже, в XX веке, американцем Штерном был создан резектоскоп - инструмент, состоящий из вольфрамовой петли, по которой пропускался электрический ток, и оптической системы, позволяющей 
проводить удаление тканей предстательной железы под контролем зрения [7]. Первая трансуретральная резекция простаты (ТУР) была выполнена в 1901 году парижским врачом Гюйоном. Однако, данный метод не сразу получил широкое распространение ввиду недостаточности научных знаний и практических умений. В течение следующего полувека последовали дальнейшие модификации, которые включали усовершенствование электрохирургической энергии, применение видеотехники, модификация рабочего элемента, линзовой и волоконно-оптической осветительной систем [17].

В настоящее время ТУР предстательной железы является «золотым стандартом» ведения пациентов с ДГПЖ. С момента своего появления ТУР простаты ассоциировалась с благоприятными исходами только при небольших размерах железы, тогда как открытая простатэктомия имела хорошие долгосрочные результаты, особенно при больших объемах гиперплазированной предстательной железы, но сопровождалась большим количеством осложнений [2]. В XX веке широкое распространение получила монополярная ТУР (мТУР), в ходе проведения которой основным осложнением оставалось кровотечение, а также ТУР-синдром, связанный с абсорбцией ирригационной жидкости в кровяное русло. В начале 2000-х годов начал развиваться метод биполярной трансуретральной резекции простаты (бТУР), принципиальным отличием которого является то, что ток проходит расстояние только между электродами, а не через все тело пациента, как это было при мТУР [4]. Проведение бТУР простаты связано со значительно меньшей частотой проявления клинически значимых осложнений, таких как ТУР-синдром и острое нарушения мочеиспускания ввиду закупорки мочевыводящих путей кровяным сгустком. Кроме того, отмечается возможность выполнения бТУР у возрастных пациентов с сопутствующей патологией [26].

Трансуретральная энуклеация предстательной железы биполярной петлей (TUEB) эффективна при удалении аденомы больших размеров, и позволяет проводить эндоскопическое вмешательство, избегая открытого хирургического доступа. TUEB целесообразно проводить при доброкачественной гиперплазии предстательной железы с объемом 80-250 см³. Способ показал аналогичную эффективность и более высокую безопасность, а также низкую частоту повторных операций в 10-летнем периоде. По данным некоторых авторов применение трансуретральной энуклеации предстательной железы возможно также при больших и гигантских размерах аденомы [11, $12,16]$.

Начиная с середины XX века, в медицинской практике стали появляться лазерные технологии. Лазер - это устройство, генерирующее интенсивный пучок когерентного монохроматического света (или другого электромагнитного излучения) за счет стимулированного излучения фотонов от возбужденных атомов или молекул. Первое зарегистрированное урологическое лазерное исследование было проведено в 1966 году, и представляло из себя влияние лазера на мочевой пузырь собаки. В 1988 году был опубликован отчет о проведении лазерной вапоризации гиперплазии предстательной железы 6 пациентам [21]. Первое применение гольмиевого лазера отмечено в 1995 году. Гольмиевая лазерная энуклеация (HoLEP) воссоздает надлобковую простатэктомию трансуретрально, и состоит из двух этапов: сначала, аналогично открытой простатэктомии, капсула предстательной железы и доли предстательной железы отделяются с помощью лазерной энергии, а затем аденома аспирируется и удаляется из мочевого пузыря [22]. Сразу же после проведения вмешательства у пациентов наблюдается значительное снижение CHMП. HoLEP характеризуется низкой вероятностью осложнений, хорошим гемостазом и полным функциональным восстановлением мочевого пузыря [18, 27]. Имеющиеся лазерные системы доказали свою клиническую эффективность, сравнимую с хорошо зарекомендовавшим себя золотым стандартом.

\section{Зәк^ючение}

ДГПж широко распространена среди мужчин преимущественно пожилого возраста, и отмечается у $90 \%$ мужчин старше 80 лет. Данная патология ощутимо снижает качество жизни пациента вследствие проявлений симптомов нижних мочевых путей. Выбор метода хирургического вмешательства во многом зависит от размера гиперплазированной предстательной железы, факторов риска и сопутствующих заболеваний. Использование современных эндовидеохирургических вмешательств, таких как трансуретральная резекция биполярной петлей, биполярная трансуретральная энуклеация, гольмиевая лазерная энуклеация, позволяет избежать большей части интра- и постоперационных осложнений, уменьшить время реабилитации пациента и количество койко-дней, проведенных пациентом в стационаре.

\section{ЛИТЕРАТУРА}

1. Безруков Е.А., Рапопорт Л. М., Морозов А. О., Мартиросян Г. А., Стрижова М. А. Эволюция техники выполнения и роли радикальной простатэктомии //Сибирское медицинское обозрение. 2017. № 3(105). С. 61-67.

2. Бершадский Я.В., Исайкин В. Г., Сангинов А. Р. Пятилетний опыт лечения доброкачественной гиперплазии предстательной железы с применением биполярной техники трансуретральных операций// Оригинальные исследования. 2019. Т. 9. № 7. С. 21-30. 
3. Данилов В.В., Осинкин К.С., Севрюков Ф. А. Воспроизводимость результатов урофлоуметрии у пациентов с аденомой предстательной железы // Вопросы урологии и андрологии. 2019. Т. 7. № 3. С. 5-9.].

4. Зубков И.В., Головизнин Ю. В., Битеев В.Х., Балдин А. В. Сравнительный анализ результатов лечения при применении монополярной и биполярной ТУР аденомы предстательной железы //Оригинальные исследования. 2017. Т. 7. № 4. С. $22-29$.

5. Зубков Э.А., Зубков А. Ю., Ситдыков Э. Н. Профилактика обструктивных осложнений чреспузырной аденомэктомии// Медицинский вестник Башкортостана. 2013. Т. 8. № 3. С. 41-44.

6. Зубков Э.А., Ситдыкова М. Э. Профилактика осложнений чреспузырной аденомэктомии с глухим швом мочевого пузыря // Казанский медицинский журнал. 2012. № 1. С. 56-61

7. Мустафаев А.Т., Кызласов П.С., Дианов М. П., Мартов А. Г., Ергаков Д. В., Севрюков Ф. А. Хирургическое лечение доброкачественной гиперплазии предстательной железы: прошлое и настоящее // Урологические ведомости. 2019. Т. 9. № 1. С. 47-56.

8. Николаев М. А. Дифференцированное использование хирургических методов в лечении пациентов с доброкачественной гиперплазией предстательной железы // International Journal of Medicine and Psychology. 2019. Т. 2. № 3. С. 89-94.

9. Севрюков Ф. А. Трансуретральная резекция в физиологическом растворе // Урология. 2012. № 2. С. 67-70.

10. Севрюков Ф.А., Малинина 0. Ю. Новые организационные технологии оказания медицинской помощи больным с доброкачественной гиперплазией предстательной железы // Социальные аспекты здоровья населения. 2012. Т. 23. № 1. С. 1-7

11. Севрюков Ф.А., Накагава К., Кочкин А. Д., Володин М. А., Семенычев Д. В. Случай успешной плазменной трансуретральной энуклеации аденомы простаты размером 530 см3 // Урология. 2019. № 2. С. 59-63.

12. Севрюков Ф.А., Nakagawa К. Использование биполярной трансуретральной энуклеации для лечения доброкачественной гиперплазии простаты больших размеров // Современные технологии в медицине. 2012. № 3. С. 46-49.

13. Сорокин Д.А., Семенычев Д. В., Володин М. А. Лечение и профилактика осложнений трансуретральных эндоскопических операций по поводу доброкачественной гиперплазии простаты // International Journal of Medicine and Psychology. 2019. Т. 2. № 4. С. 118-125.

14. Старцев В.Ю., Дударев В. А., Севрюков Ф. А., Забродина Н. Б. Экономические аспекты лечения больных с нарушениями мочеиспускания, обусловленными доброкачественной гиперплазией предстательной железы // Урология. 2019. № 6. С. 115-119.

15. Ткачук В.Н., Ткачук И. Н. История урологии в Санкт-Петербурге // Урологические ведомости. 2012. № 2. С. 3-9.

16. Arcaniolo, D., Manfredi, C., Veccia, A. et al. Bipolar endoscopic enucleation versus bipolar transurethral resection of the prostate: an ESUT systematic review and cumulative analysis. World J Urol (2019). https://doi.org/10.1007/s00345-019-02890-9

17. Babayan R.K. (2018) Development of Transurethral Resection of the Prostate (TURP). In: Patel S., Moran M., Nakada S. (eds) The History of Technologic Advancements in Urology. Springer, Cham

18. Becker, B., Gross, A.J. \& Netsch, C. Safety and efficacy using a low-powered holmium laser for enucleation of the prostate (HoLEP): 12-month results from a prospective low-power HoLEP series. World J Urol 36, 441-447 (2018). https://doi.org/10.1007/s00345-017-2159-5

19. Cho A., Chughtai B. \& Te A. E. Benign Prostatic Hyperplasia and Male Lower Urinary Tract Symptoms: Epidemiology and Risk Factors. Curr Bladder Dysfunct Rep (2020). https://doi.org/10.1007/s11884-019-00566-z

20. De Nunzio C., Salonia A., Gacci M. et al. Inflammation is a target of medical treatment for lower urinary tract symptoms associated with benign prostatic hyperplasia. World J Urol (2020). https://doi.org/10.1007/s00345-020-03106-1

21. Ingimarsson J.P., Krambeck A. E. (2018) History and Development of Lasers in the Treatment of BPH. In: Patel S., Moran M., Nakada S. (eds) The History of Technologic Advancements in Urology. Springer, Cham

22. Lei Yin, Jingfei Teng, Chien-Jung Huang, Xiangmin Zhang, and Danfeng Xu. Journal of Endourology.May 2013.604-611. http://doi.org/10.1089/end.2012.0505

23. Miner M. M. Primary care physician versus urologist: How does their medical management of LUTS associated with BPH differ? Curr Urol Rep 10, 254-260 (2009). https://doi.org/10.1007/s11934-009-0042-7

24. Novara G., Ficarra V., Zattoni F. (2014) Medical Treatment of LUTS/BPH. In: Chapple C., Tubaro A. (eds) Male LUTS/BPH Made Easy. Springer, London

25. Ozayar A., Zumrutbas A. E. \& Yaman 0. The relationship between lower urinary tract symptoms (LUTS), diagnostic indicators of benign prostatic hyperplasia (BPH), and erectile dysfunction in patients with moderate to severely symptomatic BPH. Int Urol Nephrol 40, 933-939 (2008). https://doi.org/10.1007/s11255-0089386-z

26. Tang Yin et al. (2014) "Bipolar transurethral resection versus monopolar transurethral resection for benign prostatic hypertrophy: a systematic review and metaanalysis." Journal of endourology, 28(9), 1107-14. doi:10.1089/end.2014.0188

27. Van Rij S., Gilling P. J. In 2013, Holmium Laser Enucleation of the Prostate (HoLEP) May Be the New 'Gold Standard'. Curr Urol Rep 13, 427-432 (2012). https://doi. org/10.1007/s11934-012-0279-4

28. Young M.J., Elmussareh M., Morrison T., Wilson J. R. The changing practice of transurethral resection of the prostate. Ann R Coll Surg Engl 100(4):326-329

(c) Малыхина Анастасия Сергеевна, Болгов Евгений Николаевич ( peregal@yandex.ru). Журнал «Современная наука: актуальные проблемы теории и практики» 